\title{
Enhanced bioavailability of nerve growth factor with phytantriol lipid-based crystalline nanoparticles in cochlea
}

This article was published in the following Dove Press journal:

International Journal of Nanomedicine

3 November 2015

Number of times this article has been viewed

\section{Meng $\mathrm{Bu}^{1,2}$ \\ Jingling Tang ${ }^{3}$ \\ Yinghui $\mathrm{Wei}^{4}$ \\ Yanhui Sun' \\ Xinyu Wang' \\ Linhua $\mathrm{Wu}^{2}$ \\ Hongzhuo Liu'}

'School of Pharmacy, Shenyang Pharmaceutical University, Shenyang, People's Republic of China; 2Department of Pharmacy, the Second Affiliated Hospital, ${ }^{3}$ School of Pharmacy, Harbin Medical University, Harbin, People's Republic of China; ${ }^{4}$ College of Pharmaceutical Science, Zhejiang Chinese Medical University, Hangzhou, People's Republic of China

Correspondence: Hongzhuo Liu School of Pharmacy, Shenyang Pharmaceutical University, No 103 Wenhua Road, Shenyang II0016, People's Republic of China

Tel +86242398 6258

Fax +862423986293

Email bby_1979@163.com

Linhua Wu

Department of Pharmacy, the Second Affiliated Hospital, Harbin Medical University, No 157 Baojian Road, Harbin 150086, People's Republic of China

Tel +8645 I 8660558 I

Fax +86 45I 86665559

Email yaoji636@hotmail.com
Purpose: Supplementation of exogenous nerve growth factor (NGF) into the cochlea of deafened animals rescues spiral ganglion cells from degeneration. However, a safe and potent delivery of therapeutic proteins, such as NGF, to spiral ganglion cells remains one of the greatest challenges. This study presents the development of self-assembled cubic lipid-based crystalline nanoparticles to enhance inner ear bioavailability of bioactive NGF via a round window membrane route.

Methods: A novel nanocarrier-entrapped NGF was developed based on phytantriol by a liquid precursor dilution, with Pluronic ${ }^{\circledR}$ F127 and propylene glycol as the surfactant and solubilizer, respectively. Upon dilution of the liquid lipid precursors, monodispersed submicron-sized particles with a slight negative charge formed spontaneously.

Results: Biological activity of entrapped NGF was assessed using pheochromocytoma cells with NGF-loaded reservoirs to induce significant neuronal outgrowth, similar to that seen in free NGF-treated controls. Finally, a 3.28-fold increase in inner ear bioavailability was observed after administration of phytantriol lipid-based crystalline nanoparticles as compared to free drug, contributing to an enhanced drug permeability of the round window membrane.

Conclusion: Data presented here demonstrate the potential of lipid-based crystalline nanoparticles to improve the outcomes of patients bearing cochlear implants.

Keywords: nerve growth factor, lipid-based crystalline nanoparticles, PC12 cells, inner ear drug delivery, bioavailability

\section{Introduction}

Nerve growth factor (NGF) is a native biological macromolecule that is the first and best characterized of the neurotrophin family. In degenerative diseases, neurotrophins are responsible for both the nutritional and differentiating activity of neurons in the central and peripheral nervous systems by means of protective and/or reproductive effects. ${ }^{1,2}$ It has been observed that intracerebral administration of NGF results in favorable outcomes in the treatment of Alzheimer's and Parkinson's disease. ${ }^{3,4}$ Moreover, ocular delivery of NGF accounted for the inhibition of retinal ganglion cell degeneration after mechanical, ischemic, and hypertensive injury. ${ }^{5,6}$ Neurotrophins, including NGF, brain-derived neurotrophic factor (BDNF), neurotrophin-3, and neurotrophin-4/5 have been found to play an important role in the auditory system, supporting spiral ganglion neuron survival following injury. Consequently, neurotrophins are considered to be potential therapeutic agents for improving the efficacy of the cochlear implant by enhancing spiral ganglion cell (SGC) survival in deafness. ${ }^{7,8}$

Preventing degeneration of SGCs through the delivery of neurotrophins presents a significant challenge. Failure of neurotrophins to travel across blood-cochlear barriers has resulted in an inability of these molecules to be administered systemically. 
Thus, local drug delivery of neurotrophins has generated considerable interest. The anatomy of the cochlea provides several options for neurotrophin entrance, including direct injection into the cochlea through the scala tympani, scala vestibule, or scala media, or travel across the round window membrane (RWM) into the cochlea. ${ }^{7}$ When compared to the direct injection method, RWM administration is a less invasive technique and is becoming a primary approach for effective local delivery to the inner ear. However, this technique has not been widely employed clinically because controlled and targeted drug-delivery systems have not yet been developed. ${ }^{9}$

Recently, nanoparticles have been widely used as drugdelivery vehicles due to their high capability to overcome various physiological barriers and controlled or targeted delivery profiles. When bulk, hydrated amphiphiles of bicontinuous lyotropic liquid crystalline cubic phase are dispersed in the presence of a stabilizer, nanostructured particles with remaining space structures known as cubosomes may form. Normally, cubosomes with a diameter between 100 and $200 \mathrm{~nm}$ are mechanically rigid, structurally stable, and can be biodegraded in vivo. In addition, cubosomes can accommodate significant amounts of hydrophilic, amphiphilic, and/ or lipophilic molecules due to their large interfacial surface area per unit volume, making them suitable candidates for employment as drug-delivery vehicles. ${ }^{10,11}$ Recently, using cubosomes as novel nanocarriers for protein molecules has garnered considerable interest due to their high encapsulation ability, which results in enhanced protein concentration on target sites. ${ }^{12-15}$ Our previous work demonstrated that cubosomes stabilize the earthworm fibrinolytic enzyme - a model protein. This, in turn, enhances inner ear bioavailability after intratympanic injection. ${ }^{12}$

The aim of this study was to prepare bovine serum albumin (BSA)/NGF-loaded cubosomes to improve drug delivery through the RWM to the inner ear. BSA is used as a protective barrier that occupies the cubosome interface and shields the therapeutic proteins from contact with hydrophobic surfaces, which in turn protects NGF within the delivery system. The nanostructure of the phytantriol (PHY) dispersions was determined to be cubic phase using small-angle $\mathrm{X}$-ray scattering (SAXS) and cryogenic transmission electron microscopy (cryo-TEM). The biological activity of NGF after encapsulation within the cubosome was confirmed through its ability to differentiate pheochromocytoma cells (PC12) into a neuronal phenotype. Furthermore, the pharmacokinetic profiles of NGF-encapsulated cubosomes after RWM administration were also investigated. Data presented here demonstrate the potential of lipid-based crystalline nanoparticles as a promising therapeutic treatment for sensorineural hearing loss.

\section{Materials and methods Materials}

PHY (3,7,11,15-tetramethyl-1,2,3-hexadecanetriol) was purchased from TCI (Tokyo, Japan). Pluronic F127, with an average molecular weight of 12,500 Da, was purchased from BASF (Ludwigshafen, Germany). Propylene glycol, Dimethyl Sulfoxide (DMSO), and chloroform were purchased from Yuwang Industrial Co. Ltd (Yucheng, People's Republic of China). alamarBlue was bought from Hanpu medical and biological research institute (Beijing, People's Republic of China). The $\beta$ III tubulin monoclonal antibody and donkey anti-mouse IgG were purchased from Hejun Biotechnology Co. Ltd. (Beijing, People's Republic of China). Recombinant human $\beta$-NGF and 2.5S mouse NGF were purchased from Peprotech (Rocky Hill, NJ, USA) and R\&D Systems, Inc. (Minneapolis, MN, USA), respectively. BSA was purchased from Beyotime Institute of Biotechnology (Shanghai, People's Republic of China).

\section{Preparation of formulations}

The cubosome dispersions were prepared using a dilution method as reported previously. ${ }^{13}$ Briefly, $10 \mathrm{mg}$ PHY was co-solubilized with $2.5 \mathrm{mg}$ Pluronic ${ }^{\circledR}$ F127 in chloroform. The mixture was vortexed thoroughly, followed by evaporation of the chloroform overnight under vacuum at $50^{\circ} \mathrm{C}$. The mixture was then dissolved in $26.8 \mathrm{mg}$ propylene glycol, and then $0.5 \mathrm{~mL}$ of $20 \mu \mathrm{g} / \mathrm{mL}$ NGF solution containing BSA was added at $50^{\circ} \mathrm{C}$ under constant stirring. Recombinant human $\beta$-NGF was used for all experiments except the NGF bioactivity assay.

\section{Physicochemical characterization of dispersions}

Particle-size analysis and zeta potential

A Zetasizer Nano-ZS (Malvern Instruments, Malvern, UK) was used to determine the particle hydrodynamic diameter, polydispersity index (PDI), and dispersion zeta potential. Samples were diluted with deionized water to adjust the signal level for the measurement of particle size and zeta potential, respectively. All measurements were performed in triplicate at $25^{\circ} \mathrm{C}$.

\section{Synchrotron SAXS}

SAXS measurements were carried out in order to determine the self-assembled structure of the nanostructured 
dispersions. Data were collected using the SAXS beamline at the Beijing synchrotron with a beam wavelength of $1.54 \AA$, operating at a typical flux of approximately $10^{13}$ photons/s. A Dectris-Pilatus was used to record 2D diffraction patterns with a $1.84 \mathrm{M}$ detector of ten modules. The samples were loaded into a small sample cell consisting of two very thin mica windows separated by a $1 \mathrm{~mm}$ stainless steel spacer ring and loaded into a custom built sample holder within the SAXS instrument chamber. A silver behenate with a $d$ spacing value of $58.38 \AA$ was used for a standard to calibrate the measured intensity of the angular scale. The diffraction patterns were recorded at $25^{\circ} \mathrm{C}$ for 300 seconds. SAXS data were then analyzed using the fit2D software package.

The lattice parameters $(a)$ of the mesophases were determined using:

$$
\frac{2 \pi}{q}=a \sqrt{\left(h^{2}+k^{2}+l^{2}\right)}
$$

from linear fits of the plots of $\frac{2 \pi}{q}$ versus $\sqrt{\left(h^{2}+k^{2}+l^{2}\right)}$. Here, $q$ is the measured peak position, and $h, k$, and $l$ are the Miller indices. ${ }^{16-18}$

\section{cryo-TEM}

For cryo-TEM studies, a sample droplet of $2 \mu \mathrm{L}$ was applied on a lacey carbon film-covered copper grid. Most of the liquid was then removed with blotting paper, leaving a thin film stretched over the lace holes. The specimens were instantly shock frozen by rapid immersion into liquid ethane and cooled to approximately $90 \mathrm{~K}$ by liquid nitrogen in a temperature-controlled freezing unit. After the specimens were frozen, the remaining ethane was removed using blotting paper. The specimen was inserted into a cryo-transfer holder and transferred to an FEI Tecnai 20 transmission electron microscope equipped with a Gatan UltraScan 894 CCD digital camera. Samples were viewed at a constant temperature of around $90-100 \mathrm{~K}$ and operated at $120 \mathrm{kV}$ acceleration voltage.

\section{Encapsulation efficiency of protein in PHY-based dispersions}

With the aim to quantify BSA or NGF encapsulation in cubosomes after production, $0.5 \mathrm{~mL}$ of the dispersion containing proteins was transferred into the upper chamber of a centrifuge tube fitted with an ultrafilter (Millipore Amicon ${ }^{\circledR}$ Ultra, MWCO 1,000 kD). After centrifuging the dispersion at 3,000 rpm for 10 minutes, the filtrate containing free protein was removed. The amount of protein encapsulated in the cubosomes was calculated by subtracting the amount of protein in the filtrate from the total protein. The concentration of free BSA or NGF was determined by bicinchoninic acid (Beyotime Institute of Biotechnology) and ELISA assay (Mini Human $\beta$-NGF ELISA Development Kits, Peprotech ${ }^{\circledR}$, Lot\# 1211060-M), respectively.

The drug encapsulation efficiency (EE\%) was calculated using the following equation:

$$
\mathrm{EE} \%=\frac{W_{\text {total }}-W_{\text {free }}}{W_{\text {total }}} \times 100 \approx \frac{C_{\text {total }}-C_{\text {free }}}{C_{\text {total }}} \times 100
$$

In this equation, $W_{\text {total }}$ is the total amount of protein in the cubosomes, $W_{\text {free }}$ is the amount of protein in the filtrate, $C_{\text {total }}$ is the drug concentration in the cubosomes, and $C_{\text {free }}$ is the drug concentration in the filtrate.

\section{Cell culture}

L929 cells and PC12 cells were purchased from American Type Culture Collection (ATCC Distributor Beijing Zhongyuan Ltd., Beijing, People's Republic of China) and used in the biological assay of PHY-based dispersions. The L929 cells were cultured in Eagle's Minimum Essential Medium supplemented with $10 \%$ bovine serum, whereas PC12 cells were cultured in high-glucose Dulbecco's Modified Eagle's Medium containing 10\% horse serum and 5\% fetal bovine serum. Both cell lines were kept in a $5 \% \mathrm{CO}_{2}$ humidified atmosphere at $37^{\circ} \mathrm{C}$.

\section{Cell viability}

L929 cells and PC12 cells were used for the viability studies. Cells were plated in 96-well plates at a density of $1 \times 10^{4}$ cells per well. Twenty-four hours after plating, the cells were treated with $10 \%$ DMSO (negative control), or PHY-based dispersions at lipid concentrations of $0 \mu \mathrm{g} / \mathrm{mL}$ (positive control), $4 \mu \mathrm{g} / \mathrm{mL}, 8 \mu \mathrm{g} / \mathrm{mL}, 20 \mu \mathrm{g} / \mathrm{mL}, 40 \mu \mathrm{g} / \mathrm{mL}$, and $200 \mu \mathrm{g} / \mathrm{mL}$. Viability was assessed 24 hours after the treatment of nanoparticles with the alamarBlue assay. First, culture medium was removed from the wells, which were then washed with phosphate-buffered saline (PBS) and $100 \mu \mathrm{L}$ media added. Then, $10 \mu \mathrm{L}$ of alamarBlue reagent was added to each well and incubated for 2 hours. Fluorescence was measured at an excitation wavelength of $530 \mathrm{~nm}$ and emission wavelength of $580 \mathrm{~nm}$.

\section{Bioactivity assay}

PC12 cells differentiate into a neuronal phenotype by extending neurites in response to bioactive NGF. ${ }^{19,20}$ Thus, to confirm 
the bioactivity of NGF after encapsulation into nanoparticles, PC12 cells were used as an evaluation system in vitro.

The cells were seeded at a density of $5.0 \times 10^{4}$ cells/well in $2 \mathrm{~mL}$ culture medium on a 24-well culture plate. Neurite outgrowth of PC12 cells was measured in a time-dependent manner. PC12 cells were treated with PHY-based dispersions encapsulating NGF or free NGF as a control. Cell morphology was evaluated under inverted light microscopy every other day. At the final time point, the cells were fixed with $4 \%$ paraformaldehyde and then washed twice in PBS for 10 minutes at room temperature. After fixation, the cells were washed three times with PBS and then permeabilized with $0.1 \%(\mathrm{v} / \mathrm{v})$ Triton X-100 in PBS followed by incubation in blocking buffer ( $2 \%$ BSA in PBS) for 1 hour. To determine neuronal morphology, a neuronal specific $\beta$ III tubulin monoclonal antibody (Hejun Biotechnology Co. Ltd) was used at a 1:100 dilution in blocking buffer. Following overnight incubation with the primary antibody at $4^{\circ} \mathrm{C}$, coverslips were washed twice with PBS and a fluorescein isothiocyanatelabeled donkey anti-mouse IgG secondary antibody (Hejun Institute of Biotechnology) was applied for 2 hours at room temperature. Coverslips were washed three times in PBS and incubated with Hoechst-PBS ( $1 \mu \mathrm{g} / \mathrm{mL}$; Sigma-Aldrich Co., St Louis, MO, USA) for 5 minutes then mounted on slides with Vectashield and stored at $4^{\circ} \mathrm{C}$ prior to image analysis. Imaging was done using an inverted fluorescent microscope (Olympus IX81; Mason Technologies, Dublin, Ireland). ${ }^{21}$ The level of neurite outgrowth was quantified by measuring the neurite length in three random images from triplicate experiments.

\section{In vivo pharmacokinetics analysis}

All animal studies were conducted in accordance with the guidelines of the Shenyang Pharmaceutical University Committee for the use and care of animals. All animal studies were approved and conducted in accordance with the guidelines of the Shenyang Pharmaceutical University Committee for the use and care of animals.

For these studies, we used healthy guinea pigs weighing between $300 \mathrm{~g}$ and $400 \mathrm{~g}$ with five animals per group and per time point. Animals were anesthetized with an intramuscular injection of ketamine (60 mg per kg body weight) and xylazine ( $4 \mathrm{mg}$ per $\mathrm{kg}$ body weight). The animals were placed in lateral decubitus exposing the retro auricular region of one ear. Following anesthesia, an incision in the retro auricular region (Figure 1A) was performed with a scalpel and iris scissors were used to expose the bulla mastoidea (Figure 1B). The bulla mastoidea was then opened with pliers (Figure 1C) and the round window niche observed. Nanoparticles were soaked in small pieces of surgical grade Gelfoam ${ }^{\circledR}$ and carefully inserted onto the round window niche. Finally, the hole in the bulla was sealed with dental cement. We sacrificed the animals at a predetermined time point and collected the cochleae and cochleae fluid by microinjection with a sharp tip from the top turn of the cochlea. Then, we measured the cochlea fluid volume (approximately 3-8 $\mu \mathrm{L}$ ), which was then diluted for the ELISA assay.

\section{Statistical analysis}

The results obtained in this study were statistically analyzed using Student's $t$-test with a 95\% confidence level $(P<0.05)$ and were reported as mean \pm standard deviation.


Figure I Surgical procedure of round window membrane administration.

Notes: (A) Guinea pigs in right lateral decubitus to approach the left ear. (B) Retro auricular incision of the skin. (C) Bulla mastoidea opening. 


\section{Results}

\section{Characterization of cubosome formations}

PHY dispersions are milky in appearance, which is commonly attributed to the cubic phase. ${ }^{22,23}$ The effect of BSA incorporation into PHY dispersions was studied by varying the concentration of BSA included during the cubosome production. Dynamic light scattering was then used to obtain the size and polydispersity of the nanoparticles followed by SAXS and cryo-TEM to assess the phase of the dispersions.

Particle diameter for PHY dispersions in the absence of proteins was $227.2 \pm 3.04 \mathrm{~nm}$ with a relatively low PDI (PDI $=0.075)$, indicating the narrow particle-size distribution. The protein molecules preferred entrapment in the water channels of cubosomes rather absorbed onto the surfaces or dissolved in the oil core of nanoparticles, accounting for no obvious changes in the particles sizes after the drug loading (Table 1). We determined an increase in polydispersity in the cubosome solution after addition of BSA when compared to the plain cubosomes. As also depicted in Table 1, the zeta potential of all prepared formulations was negative. Plain cubosomes had the most negative zeta potential of around $-20 \mathrm{mV}$, whilst BSA-containing cubosomes had zeta potentials of around $-23 \mathrm{mV}$. However, the differences were not statistically significant $(P>0.05)$.

The structural formations of the PHY-based nanoparticles were identified using synchrotron SAXS, where each phase is identified by its Bragg peak positions. ${ }^{24,25}$ SAXS curves revealed the presence of a bicontinuous cubic phase of $P n 3 m$ space group (with reflections spaced at $\sqrt{2}, \sqrt{3}$, and $\sqrt{4}$ ) with a lattice parameter of $7 \mathrm{~nm}$ in the dispersions containing 0,5 , and $10 \mathrm{mg} / \mathrm{mL}$ of BSA, confirming that the lack of change observed in Figure 2 is due to the retaining of the cubic phase as the BSA content increases. The cubosome architecture is also supported by cryo-TEM analysis (Figure 3 ). The coexistence of particles of cubosomes with unilamellar vesicles and highly ordered inner hexagonal structures was also observed by cryo-TEM in PHY dispersions (Figure 3). Unsurprisingly, vesicles were not detected in the SAXS experiments due to a relatively weak signal compared to the nonlamellar liquid crystalline particles. Otherwise, it is possible that the absence of the main vesicle peak and hexagonal structures was due to reduced formation in PHY dispersions.

The dilution process resulted in a relatively high EE\% of BSA. The results indicate that higher initial concentrations of BSA in the solution result in a higher incorporation of protein and loading capacity (Figure 4). It is recommended that NGF be diluted in a buffer containing a carrier of $0.1 \%$ BSA; therefore, the following evaluations were conducted with a loading amount of $1 \mathrm{mg} / \mathrm{mL}$ BSA, although the EE\% for BSA alone was marginal $(62.73 \% \pm 8.08 \%)$. However, with the protection of $1 \mathrm{mg} / \mathrm{mL} \mathrm{BSA}$, the EE\% of NGF was $93.81 \% \pm 7.65 \%$ (Figure 4 ).

\section{Cytotoxicity of cubosomes}

The alamarBlue assay works through the conversion of resazurin to the fluorescent resorufin by live cells. ${ }^{26}$ Figure 5 shows the viability of L929 and PC12 cells treated with PHY cubosomes at concentrations ranging from 0 to $200 \mu \mathrm{g} / \mathrm{mL}$. PHY cubosomes show an increase in cytotoxicity in L929 cells when compared to PC12 cells, respectively. Specifically, marked cytotoxicity at concentrations of $200 \mu \mathrm{g} / \mathrm{mL}$ was observed in PC12 cells, whereas a loss in cell viability was measured in L929 cells at a concentration of $40 \mu \mathrm{g} / \mathrm{mL}$. This may be due to lyotropic mesophase lipid nanoparticles interacting with the lipid bilayer of cells and resulting in membrane fusion, which leads to disruption of the membrane integrity and overall cytotoxicity.

\section{In vitro bioactivity}

To investigate the effect of the encapsulation process on protein bioactivity, PC12 cells were treated with NGF-loaded

Table I Particle size, PDI, zeta potential, and phase behavior of the investigated phytantriol lipid-based crystalline nanoparticles prepared by dilution method

\begin{tabular}{lllllll}
\hline Drug loading $^{\mathbf{a}}$ & Size $(\mathbf{n m})$ & PDI & Zeta $(\mathbf{m V})$ & Ratio of peaks $^{\mathrm{b}}$ & Structure of nanoparticles $^{\mathbf{c}}$ & Lattice parameter $^{\mathrm{d}}(\mathbf{n m})$ \\
\hline 0 & $227.2 \pm 3.04$ & $0.075 \pm 0.017$ & $-20.2 \pm 0.85$ & $2^{1 / 2}: 3^{1 / 2}: 4^{1 / 2}$ & Pn3m & 6.8 \\
$\mathrm{I}: 40$ & $220.9 \pm 2.83$ & $0.109 \pm 0.025$ & $-22.8 \pm 0.99$ & - & - & - \\
$\mathrm{I}: 20$ & $217.0 \pm 1.69$ & $0.104 \pm 0.028$ & $-23.25 \pm 1.90$ & - & - & - \\
$\mathrm{I}: 4$ & $222.2 \pm 1.77$ & $0.066 \pm 0.130$ & $-24.3 \pm 0.071$ & $2^{1 / 2}: 3^{1 / 2}: 4^{1 / 2}$ & Pn3m & 6.9 \\
$\mathrm{I}: 2$ & $215.6 \pm 1.91$ & $0.083 \pm 0.031$ & $-23.85 \pm 0.78$ & $2^{1 / 2}: 3^{1 / 2}: 4^{1 / 2}$ & Pn3m & 7.0 \\
\hline
\end{tabular}

Notes: Data presented are the mean \pm standard deviation of three independent experiments of freshly prepared sample characterized on the day of the formulation. ${ }^{a}$ Depicted the mass ratio of BSA with respect to lipid content; bepicted the relative ratio of Bragg peak positions; 'predicted on the basis of SAXS analysis; ${ }^{\mathrm{d}}$ calculated by the data of SAXS.

Abbreviations: BSA, bovine serum albumin; PDI, polydispersity index; SAXS, small-angle X-ray scattering. 


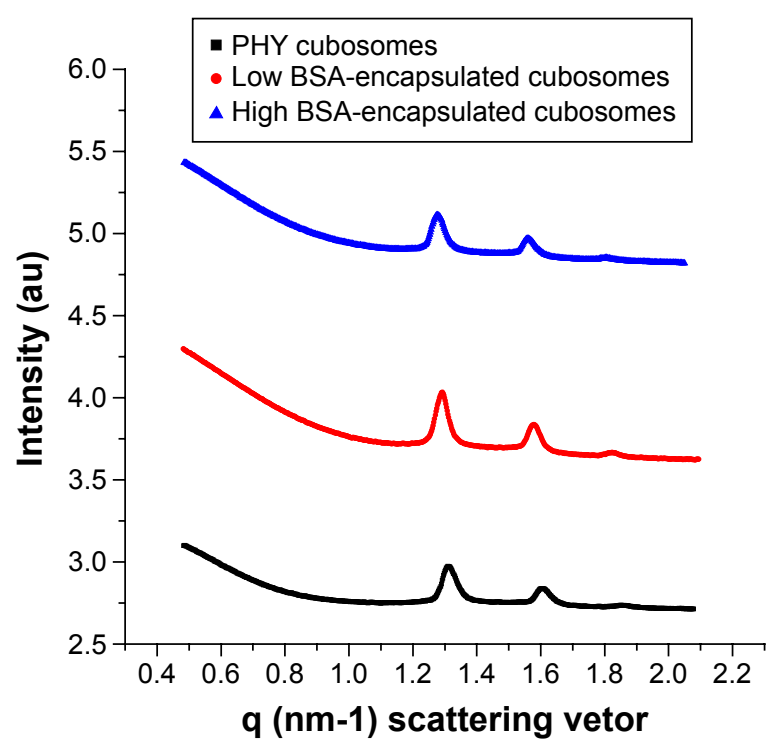

Figure 2 Small-angle $X$-ray scattering diffraction patterns of the phytantriol/FI27/ PG cubosomes which displayed typical $\mathrm{Pn} 3 \mathrm{~m}$ periodicity space group with $\sqrt{2}$, $\sqrt{3}$, and $\sqrt{4}$ Bragg reflections observed from plain cubosomes (black curve), low BSAencapsulated cubosomes (red curve, drug versus lipid mass ratio: I:4), and high BSAencapsulated cubosomes (blue curve, drug versus lipid mass ratio: I:2).

Abbreviations: BSA, bovine serum albumin; PHY, phytantriol; PG, propylene glycol.

cubosomes. PC12 cells are derived from the pheochromocytoma of a rat adrenal medulla. Therefore, the PC12 cells sprout neurites and differentiate into sympathetic ganglionlike cells in response to NGF, and thus are a useful model system of neuronal differentiation. Figure 6 shows that NGF released from cubosomes promotes neuronal cell differentiation. In contrast, PC12 cells treated with unloaded cubosomes did not differentiate and maintained round cell body shapes. Cells treated with loaded cubosomes exhibited similar morphology to NGF-treated positive controls (Figure 6A). The group administered a single dose of NGF demonstrated neuronal cell differentiation at 2 days; however, at 6 days, the cells returned to a round cell body shape similar to the untreated cells. Immunocytochemical analysis allowed for visualization of the neuron using anti$\beta$ III tubulin, a neural-specific marker (Figure 6B) that was expressed in the PC12 cells following exposure to NGFloaded cubosomes. For quantitative assessment of bioactivity in vitro, the level of neurite outgrowth was determined (Figure 6C). Specifically, the length of neurite outgrowth increased with time when PC12 cells were exposed to NGF alone or NGF-loaded cubosomes. A significant difference was observed in neuronal outgrowth of groups treated with NGF-loaded cubosomes and constant NGF treatment in comparison to single NGF-treated controls $(P<0.05)$. This may indicate that NGF-promoted differentiation of PC12 cells is reversible. Taken together, these data suggest that NGF entrapment within cubosomes and its subsequent release does not influence NGF activity.

\section{In vivo pharmacokinetics study}

NGF-loaded cubosomes were designed to improve the bioavailability of NGF. The inner ear pharmacokinetic profile of an NGF solution versus cubosome dispersions administered onto RWM is shown in Figure 7. The major pharmacokinetic
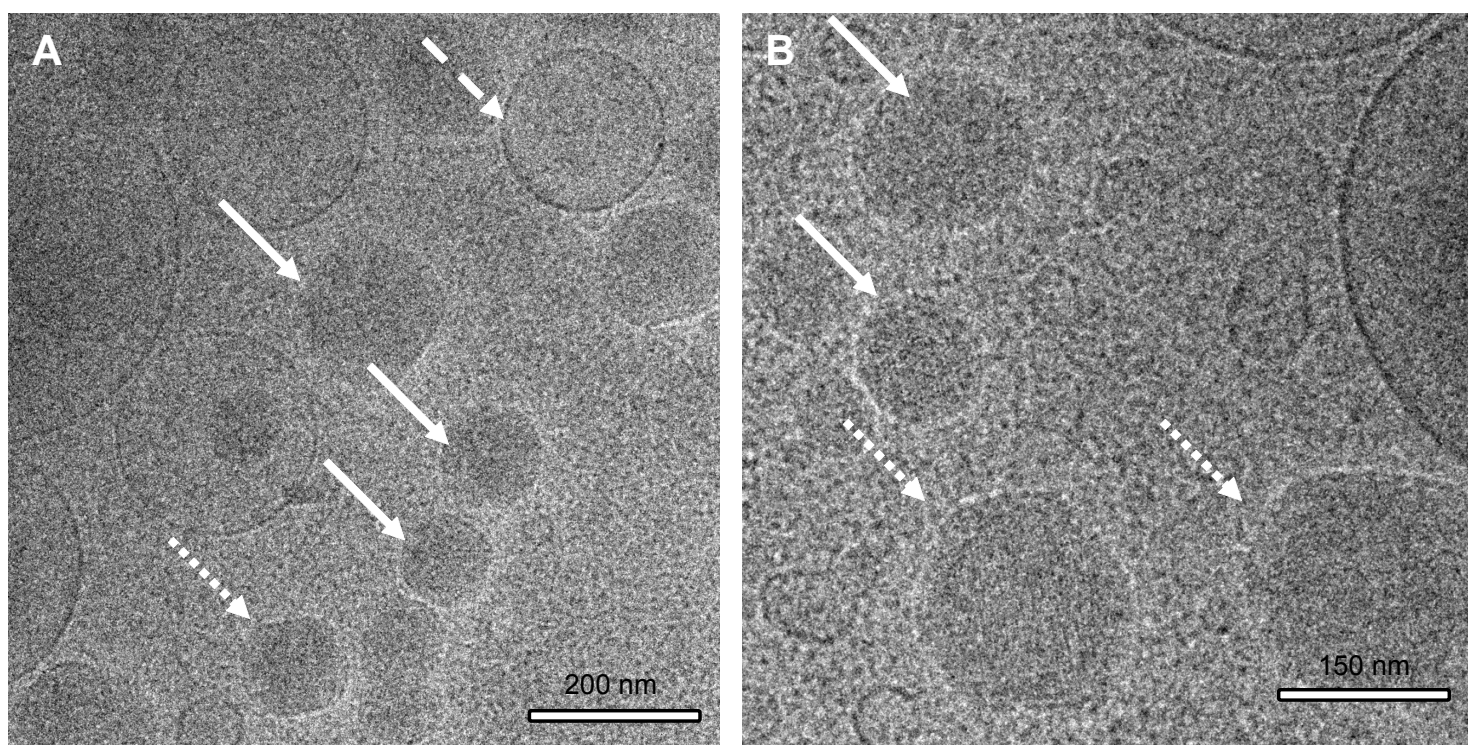

Figure 3 Cryogenic transmission electron microscopy images of the dispersed bovine serum albumin-loaded phytantriol dispersions.

Notes: (A) Overview of the dispersions showing three populations of nanoparticles: cubosomes of 100-200 nm in diameter (arrow), unilamellar vesicles of 200-500 nm in diameter (dash arrow), and (A and B) hexosomes of 100-200 nm in diameter (dot arrow). (B) Cubosomes involve cubic or micellar cubosomes, the later exhibit spheres with latticed fabric (arrow). In the case of hexosomes, they present hexagon although latticed fabric are also observed (dotted arrow). 


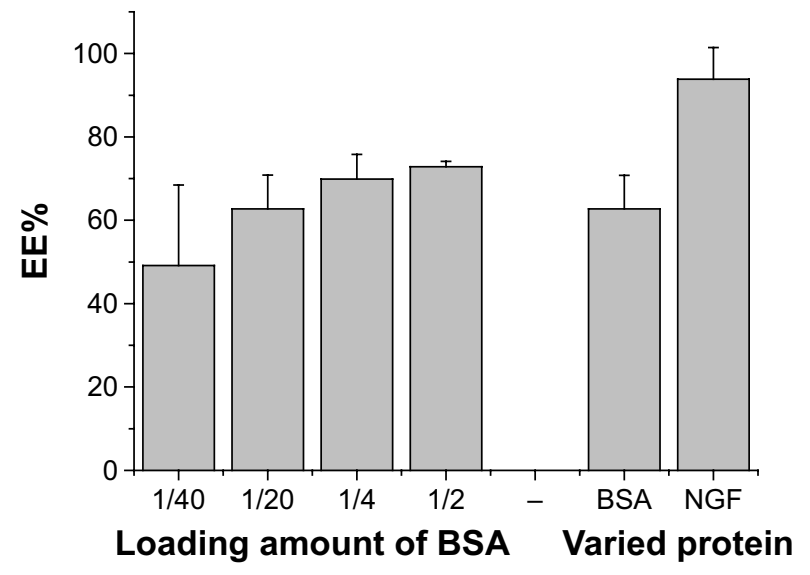

Figure 4 The EE\% of drugs in the phytantriol/FI27/PG cubosomes. Abbreviations: BSA, bovine serum albumin; EE\%, encapsulation efficiency; NGF, nerve growth factor; PG, propylene glycol.

parameters obtained are listed in Table 2, including $C_{\max }, T_{\max }$, and area under the curve (AUC) $)_{0-24 \text { hours }}$.

After treatment with free NGF, levels in the cochlear fluid peaked at 2 hours reaching a final concentration of 2,683.4 $\pm 1,149.6 \mathrm{pg} / \mathrm{mL}$. In contrast, the RWM administration of cubosomes resulted in a maximal NGF level of $13,132.5 \pm 2,528.8 \mathrm{pg} / \mathrm{mL}$ after 2 hours posttreatment. Administration of the drug using cubosomes resulted in increased distribution into the cochlear fluid at all time frames compared with that of the free drug; however, it also disappeared rapidly. Consequently, the AUC value for administration of NGF-loaded cubosomes to the RWM was significantly higher than the administration of NGF alone. In addition, the relative



Figure 5 alamarBlue assay results at 24 hours after treatment with phytantriol/ $\mathrm{FI} 27 / \mathrm{PG}$ cubosomes in L929 and PCI 2 cells.

Notes: For both cell lines, cells were treated with $4 \mu \mathrm{g} / \mathrm{mL}, 8 \mu \mathrm{g} / \mathrm{mL}, 20 \mu \mathrm{g} / \mathrm{mL}$, $40 \mu \mathrm{g} / \mathrm{mL}$, and $200 \mu \mathrm{g} / \mathrm{mL}$ nanoparticles. Data are presented as mean \pm standard deviation $(n=8)$ of percentage viability compared to untreated cells. $* P<0.05$ and ${ }^{\#} P<0.0$ I with respect to untreated groups.

Abbreviations: DMSO, Dimethyl Sulfoxide; PG, propylene glycol. bioavailability of the cubosomal formulation was increased to $328 \%$ as compared with free drug.

\section{Discussion}

In order to maintain the sense of hearing from the cochlea to the brain following cochlear implants, preservation of SGCs resulting in an extensive surviving population is required. Exogenous delivery of neurotrophic factors into the cochlea of deafened animals prevents SGCs from degenerating after the loss of hair cells. However, such information cannot be directly applied to clinical application unless appropriate delivery techniques and treatment regimes have been established. ${ }^{8}$

Intratympanic delivery of drugs to the inner ear via RWM was introduced more than half a century ago for the treatment of Ménière's disease with local anesthetics and antibiotics ${ }^{27,28}$ and has been widely used in clinics since the $1990 \mathrm{~s} .{ }^{29}$ Therefore, administration of neurotrophic factors through the RWM offers an alternative technique for less invasive delivery of therapeutic molecules to the cochlea, based upon its permeability. Although the intracochlear route of tropic agents is feasible, it has not been applied in humans because of unknown safety concerns. ${ }^{30}$

A major obstacle with respect to clinical application of neurotrophin is whether continuous treatment is required for maintaining its pharmacological function. Based on the neurotrophin hypothesis, it is believed that cessation of provided neurotrophic agents would result in the degeneration of SGCs. Evidence suggests that electrical stimulation, such as the stimulation by cochlear implants, can lead to increased survival of SGCs after loss of hair cells; however, the effects of electrical stimulation alone were found to be less than the clinically acceptable level of survival for SGCs - even when stimulation was initiated immediately after deafening. ${ }^{31,32}$ Some studies further indicate that electrical stimulation may attenuate the rapid degeneration of spiral ganglion neurons that follows withdrawal of neurotrophin therapy. For example, Maruyama et al demonstrated that SGCs survival was preserved after termination of intracochlear infusion with a glial cell line-derived neurotrophic factor in combination with the use of an electrical stimulation from a cochlear implant. ${ }^{33}$ In addition, a combination administration of BDNF and electrical stimulation with an electrically evoked auditory brainstem responses recording also resulted in enhanced survival of SGCs 2 weeks after withdrawal of the treatment and kept the SGCs from decreasing in size and circularity as observed in the untreated contralateral cochlea, indicating their synergistic effects. These studies suggest 
A

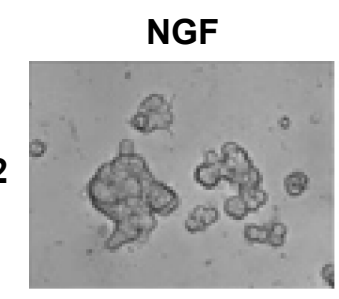

Day 2
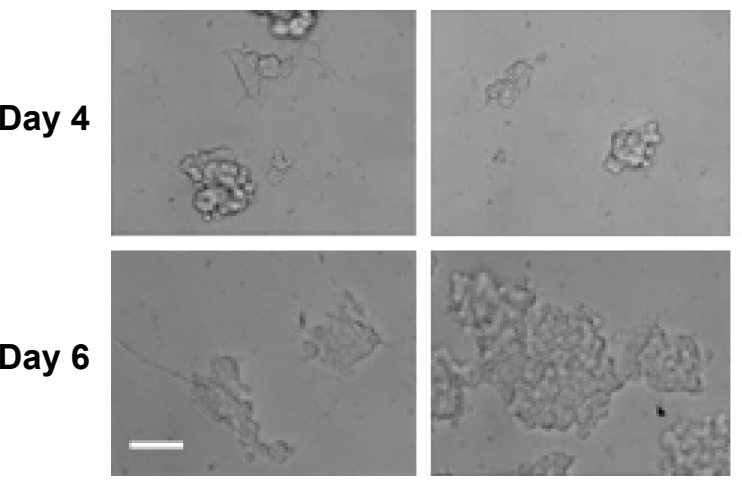

\section{B}


NGF single
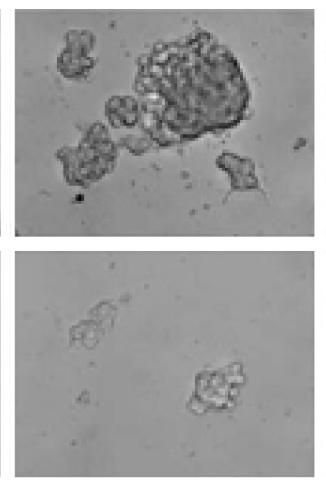

Cubosomes
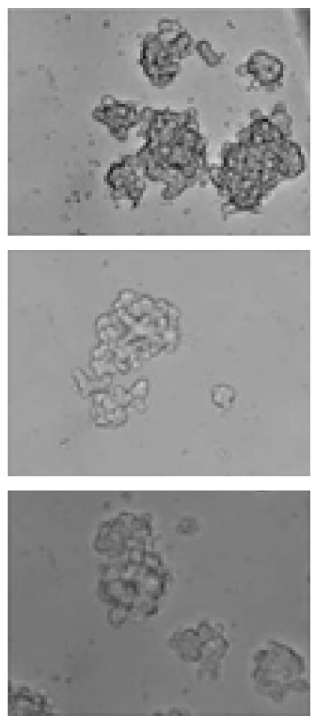

NGF cubosomes
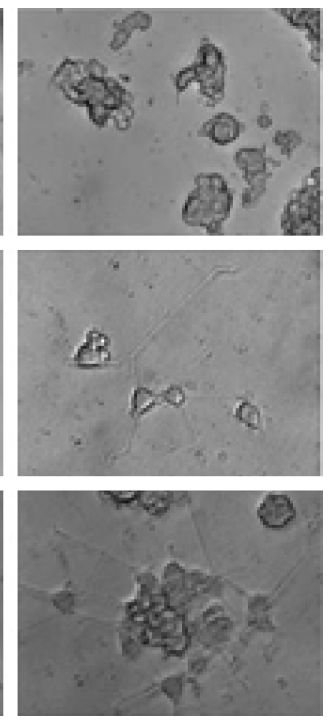

C

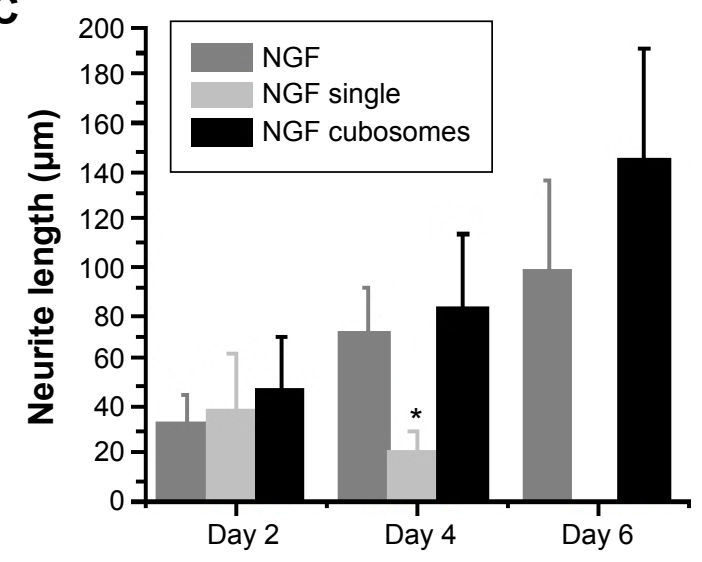

Figure 6 The bioactivity of encapsulated NGF assessed on $\mathrm{PCI} 2$ cells.

Notes: (A) PCI 2 cells treated with $10 \mathrm{ng} / \mathrm{mL}$ NGF single, or constant treatment with $10 \mathrm{ng} / \mathrm{mL} \mathrm{NGF,} \mathrm{blank} \mathrm{cubosomes,} \mathrm{or} \mathrm{NGF-loaded} \mathrm{cubosomes} \mathrm{at} \mathrm{day} \mathrm{2,} \mathrm{day} \mathrm{4,} \mathrm{and}$ day 6. (B) Neuronal outgrowth in PCI 2 cells following 6 days culture with NGF-loaded cubosomes ( $\beta$ III tubulin staining [green] of differentiated cells). Nuclei stained with Hoechst (blue) and the differentiated cells stained with $\beta$ III tubulin (green). (C) Neuronal outgrowth was observed in constant NGF treatment and in group treatment with NGF-loaded nanoparticles. Data represents mean \pm standard deviation, $n=40$ (neurites per group). $* P<0.05$ means statistical significances between NGF single and the other groups. Scale bar: $100 \mu \mathrm{m}$.

Abbreviation: NGF, nerve growth factor.

that initial, short-term neurotrophin treatment with ongoing electrical stimulation via a cochlear implant or evoked auditory brainstem responses recording might provide benefit to SGC survival. ${ }^{34}$

The application of a neurotrophin-soaked polymer or bead to the RWM resulted in preservation of SGCs throughout the cochlea, indicating the permeability of these molecules across the RWM. ${ }^{35,36}$ Based on the results of the current study, it is likely that the amount of locally applied drug entering the cochlea across the RWM may depend on the drug formulation.

The RWM is comprised of an outer epithelial layer facing the middle ear cavity, middle connection layer, and inner cellular layer facing the scala tympani perilymph. Some studies have reported that the RWM, despite being threelayers, behaves like a semipermeable membrane. However, it has been estimated that the RWM could also participate in drug absorption, metabolism, and transport based upon anatomical observations. The outer epithelium contains sparse microvilli, providing absorptive capabilities. Moreover, the outer epithelial cells have abundant organelles such as mitochondria, rough endoplasmic reticulum, and Golgi complex - indicating metabolic and transport abilities of the RWM. Based on these observations, it can be deduced that once a substance has arrived at the connective tissue layer of the RWM, it could be internalized and travel toward blood or 


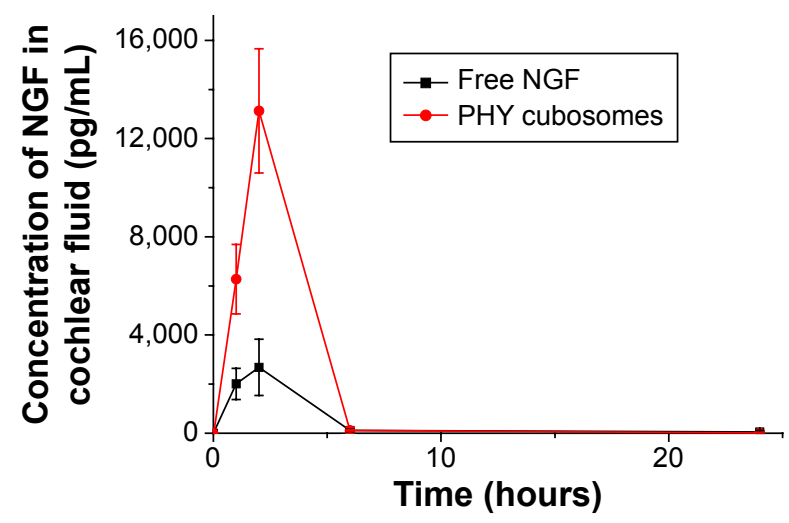

Figure 7 Cochlear concentration-time profiles of NGF after round window membrane administration.

Note: Each data point is expressed as mean \pm standard deviation $(n=6)$. Abbreviations: NGF, nerve growth factor; PHY, phytantriol.

lymph vessels. The inner epithelium of the human ear lacks continuity of the basement membranes, which provides an opening for molecules. Theoretically, a substance entering the perilymph would go through the cochlear aqueduct toward the cerebrospinal fluid and into the scala tympani or, alternatively, find its way into the endolymph. ${ }^{37}$ The permeability of the RWM therefore involves a complicated absorption, metabolism, transport, distribution, and clearance process. Consequently, the percentage of drug entering the inner ear is relatively low. It has been estimated that the cochlear fluid (perilymph) concentration of free NGF only reaches $0.009 \%$ of the applied concentration with an RWM administration protocol. Similarly, small molecules such as gentamicin or dexamethasone have been reported to reach a mean of $2.5 \%$ or $1.4 \%$ of the applied concentration when applied across the RWM. ${ }^{38,39}$ A rather low permeability for BDNF was also found when it was applied onto the RWM. ${ }^{40}$ Therefore, it can be concluded that large molecules like NGF do not easily permeate the RWM.

In the present study, nanoparticles were designed to enhance the bioavailability of NGF in the inner ear. Here, the concentration in cochlear fluid reached $0.065 \%$ of the employed drug concentration when PHY cubosomes were administered onto the RWM, which is higher than that of the free drug group (0.013\%). Correspondingly, the relative AUC value of NGF-loaded cubosomes increased significantly, although rapid clearance of NGF was observed. The cubosomes used in this investigation were a mixture of PHY and propylene glycol, which possess penetration-enhancing and moisturizing properties, respectively. It has been reported that cubosomes increase permeation of peptides into skin and nasal mucosa by providing increasingly close contact of the peptides to the cells and producing a stronger penetration with rapid extracellular transport. ${ }^{15,41}$ On the other hand, cubosomes might facilitate direct transport of NGF into the outer epithelial cells of RWM. Recent literature suggests that transcellular ${ }^{42}$ and paracellular ${ }^{43}$ pathways for nanoparticles across the RWM may occur. Transcellular pathways involve events of pinocytosis/endocytosis and subsequent exocytosis, while paracellular pathway events occur by diffusion. Whichever pathway is involved, PHY cubosomes promote the traffic of NGF into the inner ear.

As demonstrated in this study, PHY cubosomes enhance the access of NGF to the inner ear. However, the pharmacokinetic profiles of NGF in the perilymph observed after administration of PHY cubosomes and free drug were similar, indicating rapid drug absorption and clearance. Two issues might be taken into account to explain the rapid clearance. One is fast removal of drugs from the cochlear fluids via the capillary beds in the lateral wall and modiolus; the other is fast inactivation of the applied drug by metabolism. ${ }^{44}$ The latter might be the main factor leading to the reduction of NGF levels in the cochlear fluids. Nevertheless, our kinetics data indicate that enhanced NGF delivery was achieved by cubosomes as compared to free drug, indicating a promising vehicle for effective inner ear drug delivery.

\section{Conclusion}

The PHY self-assembled cubic lipid-based crystalline nanoparticles developed in this work allowed for the high encapsulation of NGF, ensuring its bioactivity. Large molecules like NGF do not easily permeate the RWM. Significantly, PHY nanoparticles enhanced the bioavailability of NGF proteins in the inner ear, suggesting a potential for the treatment of sensorineural hearing loss.

Table 2 Pharmacokinetic parameters after RWM administration of NGF or NGF-loaded phytantriol lipid crystalline nanoparticles in guinea pigs

\begin{tabular}{lllll}
\hline Formulation & $\boldsymbol{C}_{\max }(\mathrm{pg} / \mathrm{mL})$ & $\boldsymbol{T}_{\max }(\mathbf{h})$ & $\mathbf{t}_{1 / 2}(\mathbf{h})$ & AUC $_{0-24}(\mathrm{pg} / \mathrm{mL} \cdot \mathbf{h})$ \\
\hline Free NGF & $2,683.4 \pm 1,149.6$ & 2 & $7.673 \pm 5.608$ & $11,475.2 \pm 3,696.0$ \\
NGF-loaded cubosomes & $13,132.5 \pm 2,528.8$ & 2 & $12.232 \pm I 1.017$ & $37,594.8 \pm 5,403.679$ \\
\hline
\end{tabular}

Note: Values are expressed as the mean \pm standard deviation $(n=6)$.

Abbreviations: AUC, area under the curve; NGF, nerve growth factor; RWM, round window membrane; h, hour. 


\section{Acknowledgments}

This research was undertaken in part on the SAXS beamline at the Beijing synchrotron, Beijing, People's Republic of China. We thank Dr Guang Mo and Dr Zhihong Li of the Beijing synchrotron for their assistance in the setup of the SAXS beamline and further data analysis. We thank Dr Xiaojun Huang and Dr Gang Ji for technical support in cryoTEM sample preparation and data collection and gratefully acknowledge the use of the TEM facilities at the Center for Biological Imaging (CBI), Institute of Biophysics, and the Chinese Academy of Science. This study was financially supported by the National Natural Science Foundation of China (grant number 81102401) and the Program for Zhejiang Leading Team of S\&T Innovation (2012R10044_05).

\section{Disclosure}

The authors report no conflicts of interest in this work.

\section{References}

1. Aloe L, Rocco ML, Bianchi P, Manni L. Nerve growth factor: from the early discoveries to the potential clinical use. J Transl Med. 2012;10: 239.

2. Haller MF, Saltzman WM. Nerve growth factor delivery systems. J Control Release. 1998;53:1-6.

3. Cattaneo A, Capsoni S, Paoletti F. Towards non invasive nerve growth factor therapies for Alzheimer's disease. J Alzheimers Dis. 2008;15: 255-283.

4. Lad SP, Neet KE, Mufson EJ. Nerve growth factor: structure, function and therapeutic implications for Alzheimer's disease. Curr Drug Targets CNS Neurol Disord. 2003;2:315-334.

5. Lambiase A, Aloe L, Centofanti M, et al. Experimental and clinical evidence of neuroprotection by nerve growth factor eye drops: Implications for glaucoma. Proc Natl Acad Sci U S A. 2009;106:13469-13474.

6. Lambiase A, Mantelli F, Bonini S. Nerve growth factor eye drops to treat glaucoma. Drug News Perspect. 2010;23:361-367.

7. Pettingill LN, Richardson RT, Wise AK, O'Leary SJ, Shepherd RK. Neurotrophic factors and neural prostheses: potential clinical applications based upon findings in the auditory system. IEEE Trans Biomed Eng. 2007;54:1138-1148.

8. Staecker H, Garnham C. Neurotrophin therapy and cochlear implantation: translating animal models to human therapy. Exp Neurol. 2010;226: $1-5$.

9. Richardson RT, Wise AK, Andrew JK, O'Leary SJ. Novel drug delivery systems for inner ear protection and regeneration after hearing loss. Expert Opin Drug Deliv. 2008;5:1059-1076.

10. Garg G, Saraf S, Saraf S. Cubosomes: an overview. Biol Pharm Bull. 2007:30:350-353.

11. Pan X, Han K, Peng X, et al. Nanostructured cubosomes as advanced drug delivery system. Curr Pharm Des. 2013;19:6290-6297.

12. Liu H, Wang Y, Wang Q, et al. Protein-bearing cubosomes prepared by liquid precursor dilution: inner ear delivery and pharmacokinetic study following intratympanic administration. J Biomed Nanotechnol. 2013;9: 1784-1793.

13. Rizwan SB, Assmus D, Boehnke A, et al. Preparation of phytantriol cubosomes by solvent precursor dilution for the delivery of protein vaccines. Eur J Pharm Biopharm. 2011;79:15-22.

14. Shen HH, Lake V, Le Brun AP, et al. Targeted detection of phosphatidylserine in biomimetic membranes and in vitro cell systems using annexin V-containing cubosomes. Biomaterials. 2013;34:8361-8369.
15. Wu H, Li J, Zhang Q, et al. A novel small Odorranalectin-bearing cubosomes: preparation, brain delivery and pharmacodynamic study on amyloid- $\beta_{25-35}$-treated rats following intranasal administration. Eur J Pharm Biopharm. 2012;80:368-378.

16. Dong YD, Tilley AJ, Larson I, et al. Nonequilibrium effects in selfassembled mesophase materials: unexpected supercooling effects for cubosomes and hexosomes. Langmuir. 2010;26:9000-9010.

17. Murgia S, Lampis S, Zucca P, Sanjust E, Monduzzi M. Nucleotide recognition and phosphate linkage hydrolysis at a lipid cubic interface. J Am Chem Soc. 2010;132:16176-16184.

18. Warren DB, Anby MU, Hawley A, Boyd BJ. Real time evolution of liquid crystalline nanostructure during the digestion of formulation lipids using synchrotron small-angle X-ray scattering. Langmuir. 2011;27: 9528-9534.

19. Foley JD, Grunwald EW, Nealey PF, Murphy CJ. Cooperative modulation of neuritogenesis by PC12 cells by topography and nerve growth factor. Biomaterials. 2005;26:3639-3644.

20. Lin CM, Lin RD, Chen ST, et al. Neurocytoprotective effects of the bioactive constituents of Pueraria thomsonii in 6-hydroxydopamine (6-OHDA)-treated nerve growth factor (NGF)-differentiated PC12 cells. Phytochemistry. 2010;71:2147-2156.

21. Kraskiewicz H, Breen B, Sargeant T, McMahon S, Pandit A. Assembly of protein-based hollow spheres encapsulating a therapeutic factor. ACS Chem Neurosci. 2013;4:1297-1304.

22. Hartnett TE, Ladewig K, O’Connor AJ, Hartley PG, McLean KM. Size and phase control of cubic lyotropic liquid crystal nanoparticles. JPhys Chem B. 2014;118:7430-7439.

23. Muir BW, Zhen G, Gunatillake P, Hartley PG. Salt induced lamellar to bicontinuous cubic phase transitions in cationic nanoparticles. J Phys Chem B. 2012;116:3551-3556.

24. Gong X, Moghaddam MJ, Sagnella SM, et al. Lyotropic liquid crystalline self-assembly material behavior and nanoparticulate dispersions of a phytanyl pro-drug analogue of capecitabine-a chemotherapy agent. ACS Appl Mater Interfaces. 2011;3:1552-1561.

25. Negrini R, Mezzenga R. pH-responsive lyotropic liquid crystals for controlled drug delivery. Langmuir. 2011;27:5296-5303.

26. O'Brien J, Wilson I, Orton T, Pognan F. Investigation of the Alamar Blue (resazurin) fluorescent dye for the assessment of mammalian cell cytotoxicity. Eur J Biochem. 2000;267:5421-5426.

27. Ersner MS, Spiegel EA, Alexander MH. Transtympanic injection of anesthetics for the treatment of Méniére's syndrome. AMA Arch Otolaryngol. 1951;54:43-52.

28. Lange G. Gentamicin and other ototoxic antibiotics for the transtympanic treatment of Menière's disease. Arch Otorhinolaryngol. 1989;246: 269-270.

29. Blakley BW. Clinical forum: a review of intratympanic therapy. Am J Otol. 1997;18:520-526; discussion 527-531.

30. Salt AN, Plontke SK. Principles of local drug delivery to the inner ear. Audiol Neurootol. 2009;14:350-360.

31. Hansen MR, Zha XM, Bok J, Green SH. Multiple distinct signal pathways, including an autocrine neurotrophic mechanism, contribute to the survival-promoting effect of depolarization on spiral ganglion neurons in vitro. J Neurosci. 2001;21:2256-2267.

32. Leake PA, Hradek GT, Snyder RL. Chronic electrical stimulation by a cochlear implant promotes survival of spiral ganglion neurons after neonatal deafness. J Comp Neurol. 1999;412:543-562.

33. Maruyama J, Miller JM, Ulfendahl M. Glial cell line-derived neurotrophic factor and antioxidants preserve the electrical responsiveness of the spiral ganglion neurons after experimentally induced deafness. Neurobiol Dis. 2008;29:14-21.

34. Agterberg MJ, Versnel H, van Dijk LM, de Groot JC, Klis SF. Enhanced survival of spiral ganglion cells after cessation of treatment with brain-derived neurotrophic factor in deafened guinea pigs. J Assoc Res Otolaryngol. 2009;10:355-367.

35. Havenith S, Versnel H, Agterberg MJ, et al. Spiral ganglion cell survival after round window membrane application of brain-derived neurotrophic factor using gelfoam as carrier. Hear Res. 2011;272:168-177. 
36. Noushi F, Richardson RT, Hardman J, Clark G, O’Leary S. Delivery of neurotrophin-3 to the cochlea using alginate beads. Otol Neurotol. 2005;26:528-533.

37. Goycoolea MV. Clinical aspects of round window membrane permeability under normal and pathological conditions. Acta Otolaryngol. 2001;121:437-447.

38. Mikulec AA, Plontke SK, Hartsock JJ, Salt AN. Entry of substances into perilymph through the bone of the otic capsule after intratympanic applications in guinea pigs: implications for local drug delivery in humans. Otol Neurotol. 2009;30:131-138.

39. Plontke SK, Mynatt R, Gill RM, Borgmann S, Salt AN. Concentration gradient along the scala tympani after local application of gentamicin to the round window membrane. Laryngoscope. 2007;117:1191-1198.

40. Endo T, Nakagawa T, Kita T, et al. Novel strategy for treatment of inner ears using a biodegradable gel. Laryngoscope. 2005;115:2016-2020.
41. Rattanapak T, Young K, Rades T, Hook S. Comparative study of liposomes, transfersomes, ethosomes and cubosomes for transcutaneous immunisation: characterisation and in vitro skin penetration. J Pharm Pharmacol. 2012;64:1560-1569.

42. Zou J, Sood R, Ranjan S, et al. Size-dependent passage of liposome nanocarriers with preserved posttransport integrity across the middleinner ear barriers in rats. Otol Neurotol. 2012;33:666-673.

43. Zou J, Saulnier P, Perrier T, et al. Distribution of lipid nanocapsules in different cochlear cell populations after round window membrane permeation. J Biomed Mater Res B Appl Biomater. 2008;87:10-18.

44. Salt AN, Plontke SK. Local inner-ear drug delivery and pharmacokinetics. Drug Discov Today. 2005;10:1299-1306.
International Journal of Nanomedicine

\section{Publish your work in this journal}

The International Journal of Nanomedicine is an international, peerreviewed journal focusing on the application of nanotechnology in diagnostics, therapeutics, and drug delivery systems throughout the biomedical field. This journal is indexed on PubMed Central, MedLine, CAS, SciSearch $®$, Current Contents $® /$ Clinical Medicine,

\section{Dovepress}

Journal Citation Reports/Science Edition, EMBase, Scopus and the Elsevier Bibliographic databases. The manuscript management system is completely online and includes a very quick and fair peer-review system, which is all easy to use. Visit http://www.dovepress.com/ testimonials.php to read real quotes from published authors.

Submit your manuscript here: http://www.dovepress.com/international-journal-of-nanomedicine-journal 